\title{
Cirugía de contorno corporal en pacientes con pérdida masiva de peso en un hospital universitario. Estudio de frecuencias
}

\author{
Stefan Danilla E. ${ }^{1}$, Carlos Domínguez C. ${ }^{2}$, José Tomas Ganz V. ${ }^{3}$, Ekaterina Troncoso O. ${ }^{4}$, \\ Marcos Ríos V. ${ }^{4}$, Naomi Yamada T. ${ }^{5}$, Sofía Serra D. ${ }^{5}$, Patricio Andrades C. ${ }^{1}$, \\ Juan Pablo Cisternas V. ${ }^{1}$, Cristian Erazo C. ${ }^{1}$ y Sergio Sepúlveda P. ${ }^{1}$
}

\section{Body contouring surgery in patients with massive weight loss in a university hospital. Study of frequencies}

Introduction: Massive weight loss in obese, has beneficial health effects, however patients usually presents excessive adiposity and redundant skin, that generates physical and psychological problems. The body contouring surgery helps to modify the contour of the body to achieve aesthetic harmony. There are few national studies that describe the epidemiology of body contour surgery in patients with massive weight loss. Objectives: Describes a series of patients with massive weight loss with body contour surgery and its complications. Materials and Methods: A prospective series of patients with body contouring surgery with a history of low body mass, between January 2013 and June 2016, in a university hospital. Patients with incomplete information or operated at another center were excluded. Descriptive statistics was used. Results: 120 patients with body contouring surgery and low weight mass were included. Age of $38.4 \pm 10.4$ years, $105(87.5 \%)$ were women. $110(91.67 \%)$ were bariatric patients. BMI of $26.56 \pm 3.26 \mathrm{~kg} / \mathrm{m}^{2}$ and a weight loss of $39.06 \pm 14.17 \mathrm{Kg}$ were reached previous the contouring surgery. The most frequent surgery was the standard abdominoplasty $79(65.83 \%)$, followed by mastopexy with implants $21(17.5 \%) .1(0.83 \%)$ presented major complication and $33(27.5 \%)$ had a minor complication during the postoperative period. Discussion: This series presents results similar to other authors, with a lower rate of postoperative complications. Conclusion: The Body contouring surgery are safe procedures, which provides a solution to the bodily sequels after massive weight loss according to our experience. Key words: weight Loss; reconstructive surgery; postoperative complication.

\section{Resumen}

Introducción: La pérdida masiva de peso en obesos, presenta efectos beneficiosos para la salud, sin embargo esto pacientes suelen cursar exceso de adiposidad y piel redundante, generando problemas físicos y psicológicos. La cirugía de contorno corporal busca modificar el contorno del cuerpo para lograr una armonía estética. Existen pocos estudios a nivel nacional que aborden la epidemiología de la cirugía de contorno corporal en pacientes con baja masiva de peso. Objetivos: Describir una serie de pacientes con baja masiva de peso sometidos a cirugía de contorno corporal y sus complicaciones. Materiales y Métodos: Serie de casos prospectiva, de pacientes sometidos a cirugía de contorno corporal con antecedente de baja masiva de peso, entre enero de 2013 y junio de 2016, en un hospital universitario. Se excluyó a pacientes con información incompleta u operados en otro Centro. Utilizando estadística descriptiva. Resultados: 120 pacientes con cirugía de contorno corporal y baja masiva de peso, con edad de $38,4 \pm 10,4$ años, $105(87,5 \%)$ mujeres. La pérdida de peso fue en $110(91.67 \%)$ con cirugía bariátrica. Alcanzando un IMC de 26,56 $\pm 3,26 \mathrm{Kg} / \mathrm{m}^{2}$, con una pérdida de peso 39,06 $\pm 14,17 \mathrm{Kg}$. Las cirugías más frecuentes fueron la abdominoplastía estándar $79(65,83 \%)$ y la mastopexia con implantes $21(17,5 \%)$. Uno $(0,83 \%)$ presentó complicación mayor y $33(27,5 \%)$ alguna complicación menor durante posoperatorio. Discusión: Esta serie presenta resultados similares a lo descrito por otros autores, observándose una menor tasa de complicaciones en posoperatorio. Conclusiones: La cirugía de contorno corporal en este grupo de pacientes es un procedimiento seguro, que ofrece solución a las secuelas corporales pospérdida masiva de peso según nuestra experiencia.

Palabras clave: pérdida de peso; cirugía reconstructiva; complicaciones posoperatorias.
'Equipo de Cirugía Plástica y Reparadora. Departamento de Cirugía. Hospital Clínico de la Universidad de Chile.

2Fellow de Investigación en Cirugía Plástica, Departamento de Cirugía. Hospital Clínico de la Universidad de Chile. ${ }^{3}$ Hospital Clínico Padre Hurtado.

${ }^{4}$ Residente de Cirugía Plástica y Reconstructiva, Universidad de Chile.

5Interna de Medicina Universidad de Chile Santiago, Chile.

Recibido el 12 de julio de 2017, aceptado para publicación el 23 de agosto de 2017.

Correspondencia a: Dr. Stefan Danilla Enei drstefandanilla@gmail.com 


\section{Introducción}

La obesidad es actualmente una epidemia mundial ${ }^{1}$. Chile no es la excepción. La encuesta nacional de salud realizada el 2010, mostró una prevalencia de un $67 \%$ de sobrepeso. La obesidad mórbida aumentó de 148.000 habitantes/año en el 2003 a 300.000 ese mismo año (i.e., $1,3 \%$ y 3,3\% en hombres y mujeres, respectivamente) ${ }^{2}$.

La cirugía bariátrica es un tratamiento seguro y eficaz para el manejo de la obesidad, capaz de cambiar la historia natural de esta enfermedad, logrando bajas de peso significativas y sostenidas en el tiempo, así como también, mejoría de sus comorbilidades asociadas ${ }^{3,4}$. Por tal motivo, durante los últimos años, se ha visto un aumento sostenido en el número de procedimientos realizados a nivel nacional e internacional ${ }^{5}$.

Si bien los problemas de salud relacionados con la obesidad mejoran significativamente después de la pérdida de peso ${ }^{6,7}$, la capacidad de retracción de la piel no permite compensar la reducción volumétrica que sufre el cuerpo producto de la baja de peso, dejando deformidades importantes en el contorno corporal $^{8}$.

Pacientes con grandes bajas de peso suelen cursar con excedentes cutáneos significativos y formación de pliegues redundantes ${ }^{9-11}$, lo cual presenta un impacto negativo en la calidad de vida e imagen corporal de los pacientes posbariátricos pese a los beneficios de la baja de peso ${ }^{12-14}$.

La cirugía de contorno corporal ofrece estrategias reconstructivas que buscan corregir las secuelas cutáneas que se generan producto de la baja masiva de peso, siendo los procedimientos de tronco inferior, como la abdominoplastía, los más realizados ${ }^{8,15,16}$.

En la literatura internacional encontramos numerosos trabajos en que se evalúan los riesgo y resultados de pacientes con baja masiva de peso secundaria o no a cirugía bariátrica, sometidos a cirugía(s) del contorno corporal ${ }^{11,17,18}$.
Nuestro grupo de investigación ha desarrollado e implementado el instrumento PROM Body-Qo $l^{19}$, destinado a evaluar la calidad de vida de pacientes sometidos a cirugía de contorno corporal de tronco inferior, el cual ha sido utilizado para demostrar una mejora en la calidad de vida de los pacientes atribuible a la cirugía de contorno corporal tanto en pacientes con o sin antecedente de baja masiva de peso $^{20}$ y su mantención en el tiempo ${ }^{21}$.

En nuestro país existen escasos reportes sobre el manejo global e integral de los pacientes con pérdida masiva de peso, destacando lo reportado por Kauak, quien a modo de revisión del tema, presenta su experiencia consistente en 41 pacientes con antecedentes de baja masiva de peso operados de cirugía de contorno corporal entre el año 2000 y $2009^{22}$.

El objetivo del presente trabajo es describir las características sociodemográficas, clínicas, quirúrgicas y complicaciones de una serie de pacientes con baja masiva de peso sometidos a cirugía de contorno corporal y sus complicaciones.

\section{Materiales y Métodos}

Serie de casos prospectiva, obtenida del registro de la base de datos de pacientes con cirugía de contorno corporal. Se incluyó a todos los pacientes con baja masiva de peso que fueron sometidos a algún procedimiento quirúrgico de contorno corporal, entre el 01 de enero de 2013 y el 31 de junio de 2016, realizados por el equipo de cirugía plástica de un hospital universitario. Se excluyó a pacientes información incompleta u operados en otro Centro.

Este estudio fue aprobado por el Comité de Ética del hospital y realizado bajo los principios éticos propuestos en la Declaración de Helsinki ${ }^{23}$.

\section{Variables}

Se registraron variables demográficas, comorbilidades, referentes a la baja de peso, procedimientos bariátricos y cirugías de contorno corporal (Tabla 1),

Tabla 1. Procedimientos quirúrgicos destinados a mejorar el contorno corporal

\begin{tabular}{|lll|}
\hline Tronco superior & Tronco inferior & Muslos \\
Mastopexia con o sin implantes & Abdominoplastía estándar & Liposucción \\
Mamoplastía de aumento & Abdominoplastía extendida & Lifting de muslos \\
Reducción mamaria & Abdominoplastía circunferencial & \\
Braquioplastía & Abdominoplastía en flor de Lis & \\
Liposucción & Liposucción & \\
Upper Body Lift & Lower Body Lift & \\
\hline
\end{tabular}


así como complicaciones posoperatorias mayores y menores. Las variables continuas se presentan como promedio y desviación estándar, mientras que las variables categóricas, como frecuencia y porcentaje.

Se definió baja masiva de peso, como una pérdida mayor o igual al 50\% del exceso de peso.

\section{Estadística}

Se utilizó estadística descriptiva para resumir y explicar las variables. La descripción de las variables continuas se realizó mediante promedio, desviación estándar y rango. La descripción de las variables nominales se realizó mediante frecuencias y porcentajes.

Para determinar la asociación entre los factores estudiados se utilizó T test para variables continuas o normalizadas entre grupos; para variables binarias prueba de proporción z. El análisis estadístico se realizó con $S T A T A^{\circledR} 13.0$ (Stata Corp, TX, USA). Se rechazó la hipótesis nula con un nivel alfa de 1 en $20(p<0,05)$

\section{Resultados}

De un total de 1.027 pacientes intervenidos de cirugías de contorno corporal realizadas en el período analizado, $120(11,68 \%)$ correspondieron a pacientes sometidos a procedimientos del contorno corporal posterior a baja de peso masiva, los cuales presentaron una edad promedio de 38,4 $\pm 10,4$ [2064]. Ciento cinco $(87,5 \%)$ de sexo femenino con un promedio de edad de $38,84 \pm 10,2$ años y 15 $(12,5 \%)$ pacientes de sexo masculino con edades de
35,46 $\pm 11,43$ años; sin diferencias estadísticamente significativas entre ambos grupos $(p=0,236)$.

Los pacientes presentaron un Índice de Masa Corporal (IMC) basal de 41,52 $\pm 9,5 \mathrm{Kg} / \mathrm{m}^{2}$, un IMC (mínimo) $26,56 \pm 3,23 \mathrm{Kg} / \mathrm{m}^{2}$ en el momento de máxima baja de peso y un IMC 26,56 $\pm 3,26$ $\mathrm{Kg} / \mathrm{m}^{2}$ al momento de la cirugía de contorno corporal, lo cual corresponde a una disminución de $39,06 \pm 14,17 \mathrm{Kg}$ de peso.

Diez $(8,33 \%)$ pacientes lograron bajar de peso mediante cambio en el estilo de vida y $110(91,67 \%)$ asociado a cirugía bariátrica, de los cuales 56 $(50,90 \%)$ fueron gastrectomía vertical en manga y $54(49,09 \%)$ bypass gástrico.

La cirugía de contorno corporal se realizó en promedio 4,01 $\pm 2,72$ años posterior a la cirugía bariátrica (Tabla 2).

En el período evaluado se realizaron 151 cirugías de contorno corporal asociados a los 120 pacientes. De ellos, 93 (77,5\%) fueron operados una vez, 23 $(19,2 \%)$ dos, y $4(3,3 \%)$ tres veces en segmentos corporales distintos. Se realizó cirugía combinada de más de un segmento corporal en un mismo tiempo quirúrgico en $6(0,5 \%)$ casos.

Las cirugías realizadas fueron organizadas en tres segmentos corporales, tronco superior, tronco inferior y muslos.

Se realizó procedimientos destinados a corregir las secuelas de la baja masiva de peso en tronco superior a $48(40 \%)$ pacientes. En hombres (3 pacientes), se realizó liposucción de la región pectoral como manejo de pseudoginecomastia en $1(6,67 \%)$ caso y en $2(13,33 \%)$ pacientes el tratamiento del tronco superior fue realizado en forma íntegra

Tabla 2. Características epidemiológicas de la serie

\begin{tabular}{|lccc|}
\hline & $\begin{array}{c}\text { Hombres } \\
\mathbf{n = 1 5}\end{array}$ & $\begin{array}{c}\text { Mujeres } \\
\mathbf{n = 1 0 5}\end{array}$ & Valor p \\
Edad & $35,46 \pm 11,43$ & $38,84 \pm 10,26$ & 0,236 \\
IMC máximo $\left(\mathrm{Kg} / \mathrm{m}^{2}\right)$ & $46,79 \pm 6,34$ & $40,96 \pm 5,51$ & 0,001 \\
IMC mínimo $\left(\mathrm{Kg} / \mathrm{m}^{2}\right)$ & $26,03 \pm 9,06$ & $24,60 \pm 5,502$ & 0,457 \\
IMC precirugía contorno corporal & $30,05 \pm 3,04$ & $26,07 \pm 2,99$ & 0,001 \\
Pérdida de peso & $52,13 \pm 18.45$ & $37,33 \pm 12.66$ & 0,001 \\
Cirugía bariátrica & $12(80 \%)$ & $98(93,3 \%)$ & 0,014 \\
Tipo cirugía bariátrica $\quad$ & & \\
$\quad$ Bypass gástrico & $7(58,33 \%)$ & $45(45,91 \%)$ & 0,449 \\
$\quad$ Gastrectomía vertical tubular & $4(33,33 \%)$ & $49(50 \%)$ & \\
Desconocido & $1(8,33 \%)$ & $4(4,08 \%)$ & 0,622 \\
\hline
\end{tabular}




\section{ARTíCULO ORIGINAL}

mediante Upper Body Lift. En pacientes de sexo femenino se realizó cirugía de mama en $36(34,28 \%)$ casos, Upper Body Lift $6(5,71 \%)$ y braquioplastía $3(2,86 \%)$. Las cirugías de mama más frecuentes fueron mastopexia con implantes $21(58,33 \%)$, mastopexia sin implantes $7(19,44 \%)$ y mamoplastía de aumento $5(13,89 \%)$.

Las cirugías de tronco inferior se realizaron en $96(80 \%)$ pacientes, el procedimiento más realizado fue la abdominoplastía $79(65,83 \%)$ y el Lower Body Lift en 17 (14,16\%) pacientes (Figuras 1 y 2). El patrón de resección cutáneo más frecuentes fue la abdominoplastía estándar $49(62,02 \%)$ y abdominoplastía extendida $13(16,46 \%)$. No se observó diferencia estadísticamente significativa entre sexos $(\mathrm{p}=0,712)$.

Siete $(5,83 \%)$ de los pacientes fueron sometidos a procedimientos con el objetivo de mejorar el contorno de muslos: lifting de muslos en $6(5 \%)$ y liposucción en $1(8,33 \%)$ un caso. Todos los casos fueron de sexo femenino (Tabla 3 ).

La serie considera $27(22,5 \%)$ pacientes, se realizaron al menos 2 cirugías de contorno corporal, siendo la combinación más frecuente la asociación
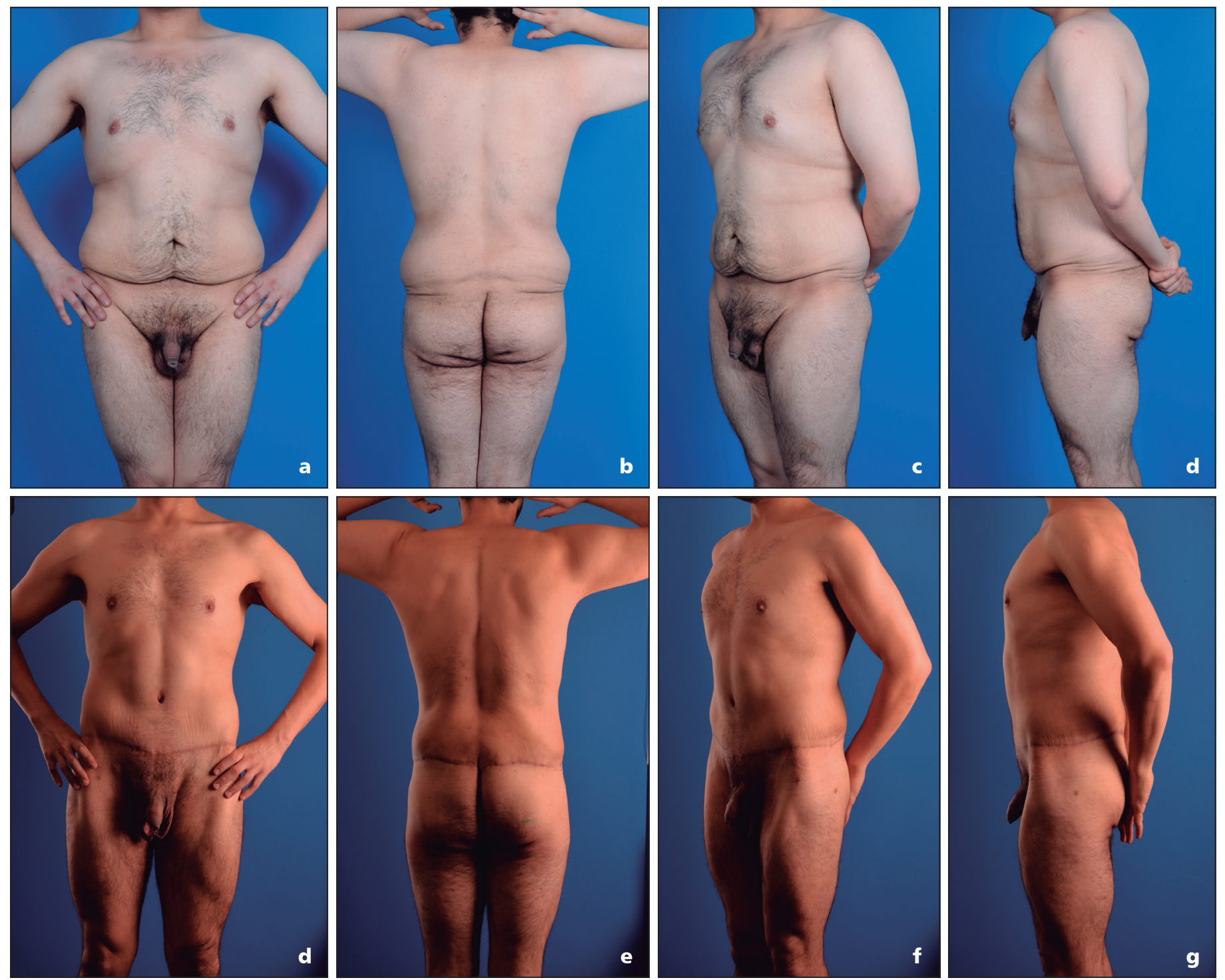

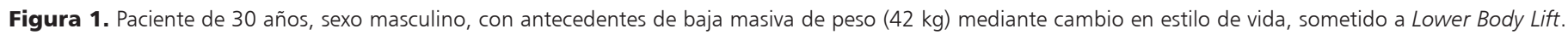
Se presenta serie fotográfica preoperatoria (a-d) y resultados posoperatorios a los 3 meses (e-h). 
de procedimiento del tronco superior e inferior 22 $(18,33 \%)$ (Figura 3$)$ y $4(3,33 \%)$ cirugía de tronco superior, inferior y muslos.

Se realizó un procedimiento concomitante en 76 $(63,33 \%)$ pacientes, siendo los más frecuentes la liposucción $51(42,5 \%)$, liposucción más lipoinyección $18(15 \%)$ y la hernioplastía en $6(5 \%)$ casos.

En relación a las complicaciones, podemos destacar que durante la hospitalización, sólo $1(0,83 \%)$ paciente presentó un troboembolismo pulmonar (complicación mayor) sin compromiso hemodinámico asociado, siendo manejado con anticoagulación oral en forma ambulatoria.
La serie presenta un seguimiento promedio de $3,37 \pm 0,97$ años, durante el cual se ha observado el desarrollo de alguna complicación menor en 33 $(27,5 \%)$ pacientes. Las más prevalentes fueron la dehiscencia de herida (Figura 4a) operatoria en 13 $(10,83 \%)$ pacientes, cicatriz inestética (Figura $4 b)$ en $12(10 \%)$ y seroma en $7(5,83 \%)$. Fue requerida la realización de una nueva intervención para manejo de sus complicaciones en $7(5,83 \%)$ pacientes (4 cicatrices intestéticas y 3 dehiscencias de herida operatoria), el resto de las complicaciones fueron manejadas en forma ambulatoria mediante curaciones. No se registró mortalidad intra ni perioperatoria.
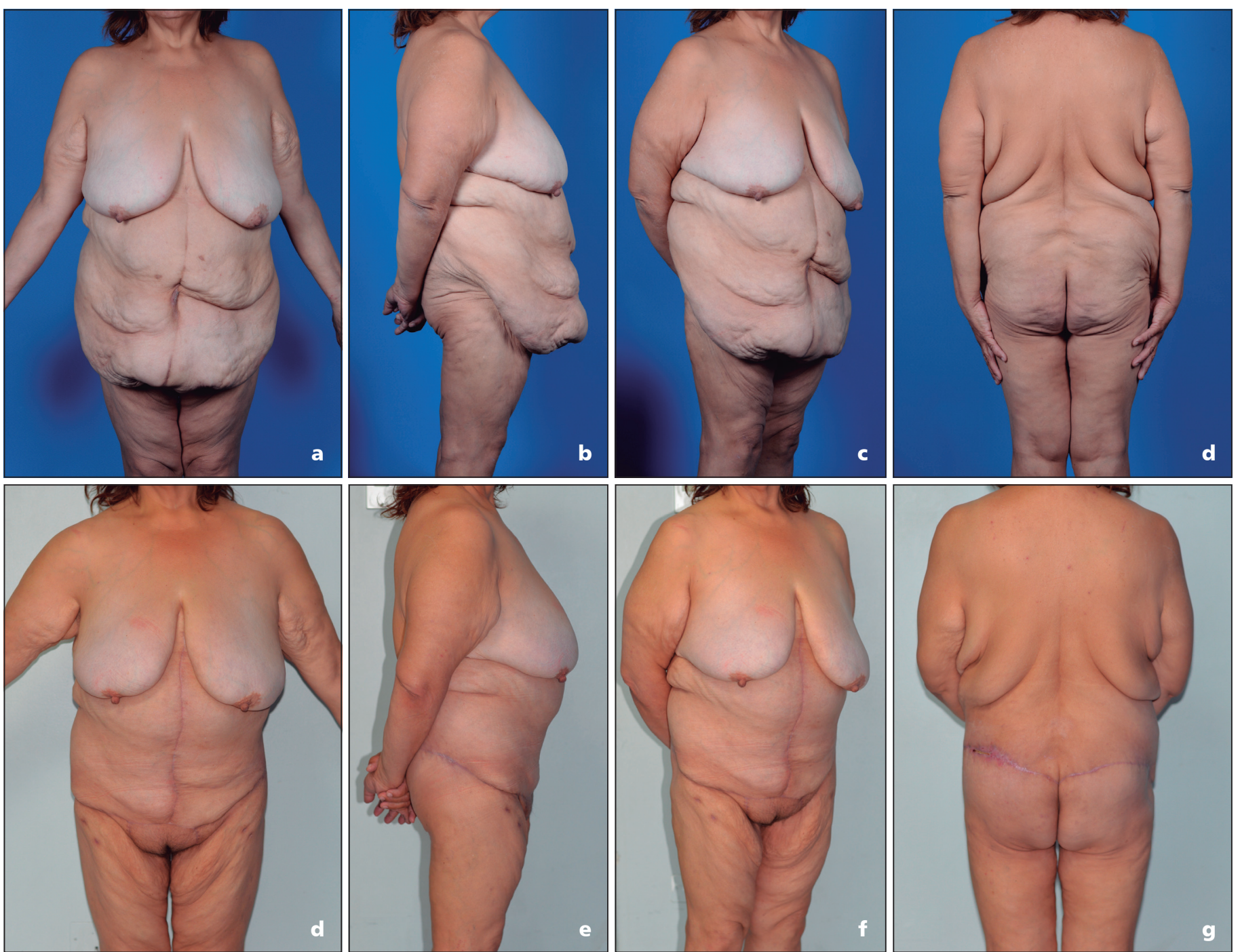

Figura 2. Paciente de 58 años, con antecedente de bypass gástrico Abierto y pérdida masiva de peso de 54 kg, sometida a Lower Body Lift (patrón de resección en flor de Lis). Se presenta serie fotográfica preoperatoria (a-d) y resultados posoperatorios a los 2 meses (e-h) 
Tabla 3. Distribución de procedimientos de contorno corporal en pacientes post baja masiva de peso

\begin{tabular}{|c|c|c|c|}
\hline Procedimiento & $\begin{array}{c}\text { General } \\
\mathbf{n}=\mathbf{1 2 0}\end{array}$ & $\begin{array}{c}\text { Hombre } \\
\mathrm{n}=15\end{array}$ & $\begin{array}{c}\text { Mujer } \\
\mathbf{n}=105\end{array}$ \\
\hline Tronco superior & $48(37,5 \%)$ & $3(20 \%)$ & $45(42,86 \%)$ \\
\hline Liposucción & $1 \quad(0,83 \%)$ & $1 \quad(6,67 \%)$ & 0 \\
\hline Braquioplastía & $3(2,5 \%)$ & 0 & $3(2,86 \%)$ \\
\hline Mastopexia con implante & $21(17,5 \%)$ & - & $21(20 \%)$ \\
\hline Mastopexia sin implante & $7(5,83 \%)$ & - & $7(6,67 \%)$ \\
\hline Reducción mamaria & $3(2,5 \%)$ & - & $3(2,86 \%)$ \\
\hline Mamoplastía aumento & $5(4,17 \%)$ & - & $5(4,76 \%)$ \\
\hline Upper Body Lift & $8 \quad(6,67 \%)$ & $2(13,33 \%)$ & $6 \quad(5,71 \%)$ \\
\hline Tronco inferior & $96(80 \%)$ & $14(93,3 \%)$ & $82(78,1 \%)$ \\
\hline Mini abdominoplastía & $1(0,83 \%)$ & $1 \quad(6,67 \%)$ & 0 \\
\hline Abdominoplastía estándar & $79(65,83 \%)$ & $5(33,33 \%)$ & $44(41,9 \%)$ \\
\hline Abdominoplastía extendida & $13(10,83 \%)$ & $4(26,67 \%)$ & $9(8,57 \%)$ \\
\hline Abdominoplastía circunferencial & $5(4,17 \%)$ & $1 \quad(6,67 \%)$ & $4(3,81 \%)$ \\
\hline Abdominoplastía en flor de lis & $11 \quad(9,17 \%)$ & 0 & $11(10,48 \%)$ \\
\hline Lower Body Lift & $17(14,17 \%)$ & $3(20 \%)$ & $14(13,33 \%)$ \\
\hline Muslos & $7 \quad(5,83 \%)$ & 0 & $7 \quad(6,67 \%)$ \\
\hline Liposucción & $1 \quad(0,83 \%)$ & 0 & $1 \quad(9,52 \%)$ \\
\hline Lifting de muslos & $6 \quad(5 \%)$ & 0 & $6 \quad(5,7 \%)$ \\
\hline
\end{tabular}

\section{Discusión}

La serie presentada, resume la experiencia acumulada en cirugía de contorno corporal por el equipo de cirugía plástica y reconstructiva del HCUCH en pacientes con baja masiva de peso. Alrededor del $11 \%$ de los pacientes que se realizan una cirugía de contorno corporal lo hacen posterior a la pérdida masiva de peso, siendo la cirugía bariátrica el principal mecanismo para bajar de peso. Series internacionales presentan porcentajes variables de pacientes posbariátricos en cirugía de contorno corporal $(7-47 \%)^{11,16,24}$, con bajas de peso de hasta $65 \mathrm{~kg}$, alcanzando un IMC precirugía de contorno corporal $30-35 \mathrm{Kg} / \mathrm{m}^{2} 9,25$, con tiempos medios hasta la cirugía de contorno corporal de entre 20 y 30 meses ${ }^{10,25}$. Lo cual difiere con los resultados presentados en esta serie donde se operaron pacientes que presentaron una menor baja de peso promedio $(52 \mathrm{Kg})$ y alcanzaron un IMC previo a la cirugía de contorno corporal $\left(26,6 \mathrm{Kg} / \mathrm{m}^{2}\right)$ cercano al límite de normalidad. Se observa, además, que los pacientes presentados en nuestra serie presentaron un mayor tiempo entre la cirugía bariátrica y de contorno corporal.

Según estadísticas de la Sociedad Americana de cirugía plástica, el $2017^{26}$, el procedimiento más frecuente en pacientes con baja masiva de peso es la abdominoplastía (39\%), seguido por la cirugía mamaria (26\%) y de brazos (13\%), presentando procedimientos más extensos (Upper y Lowe body lift) frecuencias menores al 10\%. En nuestra experiencia, si bien la abdominoplastía $(65,83 \%)$ y la cirugía de mama $(34,28 \%)$ son los procedimientos más frecuentes, cirugías extensas (Body lift) presentan una frecuencia del $20,83 \%$ lo cual refleja compromiso docente de nuestro centro en impulsar técnicas más complejas.

Por protocolo de nuestro equipo de cirugía plástica se evita realizar grandes procedimientos combinados en segmentos corporales diferentes para no prolongar los tiempos operatorios con el objetivo de disminuir las complicaciones asociadas, por tal motivo $22,5 \%$ de los pacientes fueron intervenidos en más de una oportunidad para poder lograr un tratamiento final. Solo $5 \%$ presentó procedimientos combinados (abdominoplastía con cirugía mamaria), lo cual alcanza hasta el 46-60\% según describe Zammerilla ${ }^{9}$ y Chong ${ }^{27}$.

El desarrollo de complicaciones mayores como tromboembolismo pulmonar presenta un incidencia reportada por $\mathrm{Aly}^{28}$ de $9 \%$. En nuestra experiencia, $1(0,83 \%)$ paciente presentó un evento tromboembólico. Esta baja tasa de complicaciones se podría deber al manejo protocolizado de todos los pacientes sometidos a cirugía de contorno corporal, evaluando riesgo tromboembólico, uso de heparina de bajo peso molecular, dispositivos de compresión neumática intermitente, estimular la deambulación temprana y disminución de los tiempos quirúrgi$\cos ^{29}$, además, de evitar realizar procedimientos combinados extensos y la implementación de dos equipos quirúrgicos en procedimientos extensos. Se destaca, además, que no reportó mortalidad ni otro tipo de complicaciones mayores.

El desarrollo y validación del instrumento Body. $Q o L^{\circledR}$ por nuestro equipo ${ }^{30}$, ha permitido evaluar el impacto asociado a los procedimientos de cirugía de contorno corporal en la calidad de vida de los pacientes tanto con y sin antecedente de pérdida masiva de peso $^{31}$. Nuestros resultados muestran un cambio favorable en la calidad de vida poscirugía de contorno corporal (aumento de 41,5 puntos) y que este se mantiene en el tiempo a largo plazo $(p=0,112)^{32}$. Estos resultados son concordantes con lo publicado por Klassen ${ }^{33}$ y Van der Beek ${ }^{34}$.

Las complicaciones menores presentan una incidencia reportada entre $20-70 \%$ en series internacionales $^{35,36}$, a nivel nacional describe $26,3 \%{ }^{22}$. El seroma es por lo general la complicación más común re- 


\section{ARTíCULO ORIGINAL}
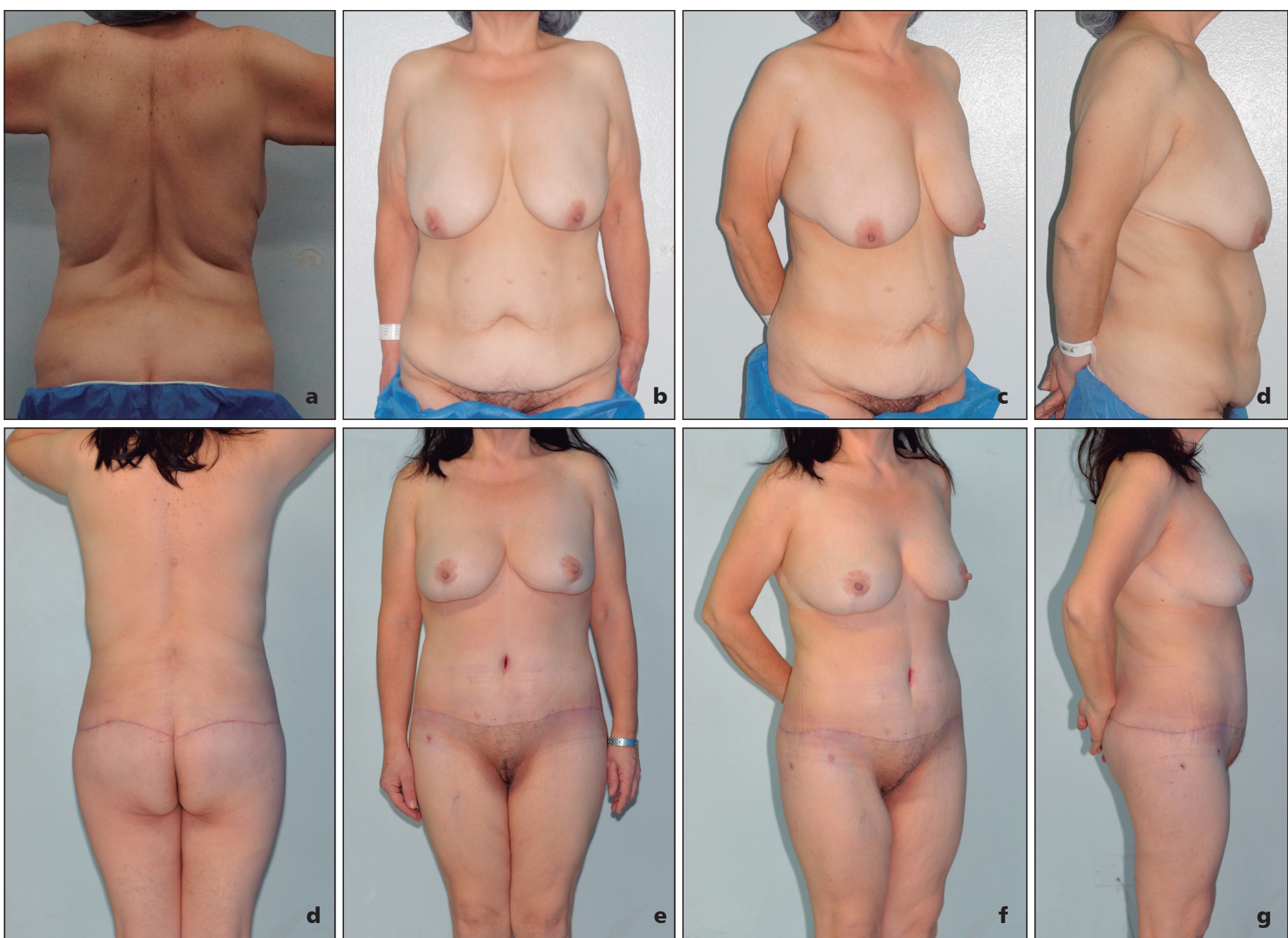

Figura 3. Paciente de 53 años, sexo femenino, con antecedente de gastrectomía tubular vertical y pérdida masiva de peso de 43 Kg. Sometida a Upper Body Lift y posteriormente Lower Body Lift para dar manejo integral al tronco superior e inferior. Se presenta serie fotográfica basales (previo a la realización de Upper Body Lift) (a-d) y resultados finales 2 meses post Lower Body Lift (e-h)
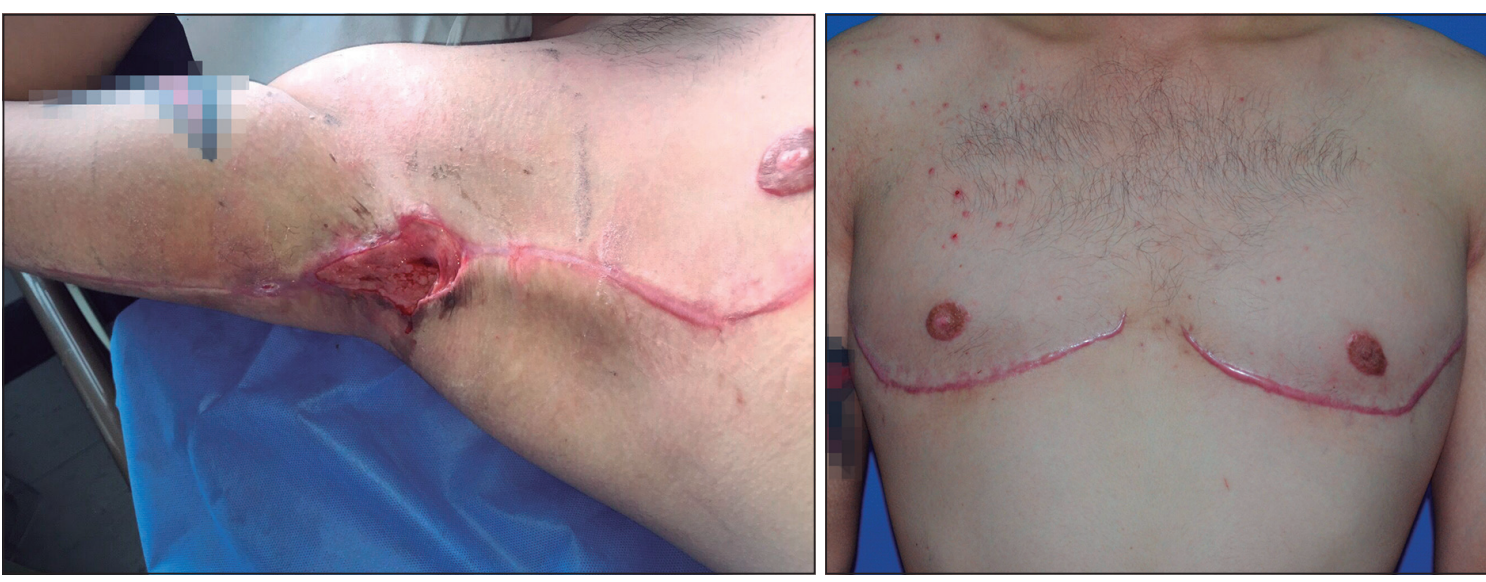

Figura 4. Complicaciones de cutáneas en cirugía de contorno corporal. (a) Dehiscencia de herida operatoria axilar en paciente con Upper Body Lift; (b) Cicatriz Inestetica en paciente con Upper Body Lift. 
portada en este tipo de cirugías, con una frecuencia $13-37 \% \%^{25,37,38}$, seguido por la dehiscencia de herida operatoria $(22-30 \%)^{39,40}$. En nuestra experiencia el desarrollo de complicaciones menores presentó una incidencia de $27,5 \%$, siendo la dehiscencia de herida operatoria la complicación más frecuente $(10,83 \%)$, seguido por la cicatriz inestética $(10 \%)$ y en tercer lugar el desarrollo de seromas $(5,83 \%)$. La baja tasa de seromas se puede deber al uso rutinario de drenajes ${ }^{41}$ y limitado en abdominoplastías. La selección adecuada, manejo preoperatorio y posoperatorio protocolizado de pacientes con baja masiva de peso, sometidos a cirugía de contorno corporal, presenta buenos resultados, siendo procedimientos seguros para los pacientes.

\section{Responsabilidades éticas}

Protección de personas y animales. Los autores declaran que para esta investigación no se han realizado experimentos en seres humanos ni en animales.

Confidencialidad de los datos. Los autores declaran que han seguido los protocolos de su centro de trabajo sobre la publicación de datos de pacientes.

Derecho a la privacidad y consentimiento informado. Los autores han obtenido el consentimiento informado de los pacientes y/o sujetos referidos en el artículo. Este documento obra en poder del autor de correspondencia.

\section{Financiación}

Este estudio no recibió subvenciones de terceros.

\section{Conflicto de intereses}

Los autores declaran no tener ningún conflicto de intereses.

\section{Agradecimiento}

A Hospital Clínico de la Universidad de Chile.

\section{Bibliografía}

1. Fountaine K, Redden D, Wang C, Westfall A, Allison D. Years of Life Lost Due to Obesity. JAMA 2003;289:187-93.

2. Ministerio de Salud. Encuesta Nacional de Salud 2009-2010 [Internet]. 2011. Available from: http://www.redsalud.gov. cl/portal/docs/page/minsalcl/g_home/ submenu_portada_2011/ens2010.pdf

3. Lee W-J, Chong K, Lin Y-H, Wei J-H, Chen S-C. Laparoscopic Sleeve Gastrectomy Versus Single Anastomosis (Mini-) Gastric Bypass for the Treatment of Type 2 Diabetes Mellitus: 5-Year Results of a Randomized Trial and Study of Incretin Effect. OBES SURG. Springer US; 2014; 24:1552-62.

4. Powell MS, Fernandez AZ Jr. Surgical Treatment for Morbid Obesity: The Laparoscopic Roux-en-Y Gastric Bypass. Surgical Clinics of NA. Elsevier Inc; 2011 Dec 1;91(6):1203-24.

5. Maluenda F. Cirugía Bariátrica. Revista Médica Clínica Las Condes. 2012;23:1808.

6. Lanzarini E, Csendes A, Gutiérrez L, Cuevas P, Lembach H, Molina JC, et al.
Type 2 Diabetes Mellitus in Patients with Mild Obesity: Preliminary Results of Surgical Treatment. OBES SURG. Springer-Verlag; 2012; 23:234-40.

7. Papapietro K, Díaz E, Díaz JC, Csendes A, Braghetto I, Burdiles P, et al. Evolución de comorbilidades metabólicas asociadas a obesidad después de cirugía bariátrica. Rev Med Chile 2005;133:5116.

8. Richter DF, Stoff A, Velasco-Laguardia FJ, Reichenberger MA. Circumferential Lower Truncal Dermatolipectomy. Clinics in Plastic Surgery. 2008;35:53-71.

9. Zammerilla LL, Zou RH, Dong ZM, Winger DG, Rubin JP, Gusenoff JA. Classifying Severity of Abdominal Contour Deformities after Weight Loss to Aid in Patient Counseling. Plastic and Reconstructive Surgery. 2014;134:888e-894e.

10. Ellison JM, Steffen KJ, Sarwer DB. Body Contouring After Bariatric Surgery. de Zwaan M, Mitchell JE, editors. Eur Eat Disorders Rev. 2015;23:479-87.

11. de Kerviler S, Hüsler R, Banic A, Constantinescu MA. Body Contouring Surgery Following Bariatric Surgery and
Dietetically Induced Massive Weight Reduction: A Risk Analysis. Obes Surg. 2008;19:553-9.

12. Song A, Rubin JP, Thomas V, Dudas J, Marra KG, Fernstrom M. Body Image and Quality of Life in Post Massive Weight Loss Body Contouring Patients. Obes Surg. 2006;14:1-11.

13. Danilla S, Cuevas P, Domínguez C, Jara R, Ríos MA, Calderón González M, et al. Body- QolL. Un nuevo instrumento de evaluación de resultados desde la perspectiva del paciente en cirugía del contorno corporal estética y post bariátrica. Rev Chil Cir. 2015;67:493-505.

14. Manterola C, Urrutia S, Otzen T. Calidad de vida relacionada con salud. Instrumentos de medición para valoración de resultados en cirugía digestiva alta*. Rev Chil Cir 2014;66:1-9.

15. Colwell AS. Current Concepts in Postbariatric Body Contouring. Obes Surg. 2010;20:1178-82.

16. Kitzinger HB, Abayev S, Pittermann A, Karle B, Kubiena H, Bohdjalian A, et al. The Prevalence of Body Contouring Surgery After Gastric Bypass Surgery. Obes Surg. 2011;22:8-12. 
17. Winocour J, Gupta V, Ramirez JR, Shack RB, Grotting JC, Higdon KK. Abdominoplasty: Risk Factors, Complication Rates, and Safety of Combined Procedures. Plastic and Reconstructive Surgery. 2015;136:597e-606e.

18. Hasanbegovic E, Sørensen JA. Complications following body contouring surgery after massive weight loss: A meta-analysis. British Journal of Plastic Surgery. Elsevier Ltd; 2014;67:295-301.

19. Danilla E, Cuevas T, Calderón ME, Ríos A, Marco V, Dominguez C, et al. Development of an instrument to evaluate the results of plastic surgery from the perspective of the patient. Rev Chil Cir. 2013;65:495-501.

20. Danilla S, Cuevas P, Aedo S, Domínguez $\mathrm{C}$, Jara R, Calderón ME, et al. Introducing the Body-QoL ${ }^{\circledR}$ : A New Patient-Reported Outcome Instrument for Measuring Body Satisfaction-Related Quality of Life in Aesthetic and Post-bariatric Body Contouring Patients. Aesthetic Plast Surg. Springer; 2016;40:19-29.

21. Troncoso E, Suijker J, Pizarro F, Montecinos S, Villarroel G, Cavada G, et al. Resultados a largo plazo en calidad de vida en cirugía de contorno corporal. Fase IV en la cohorte Body-Qol ${ }^{\circledR}$. Rev Chil Cir 2017 May;69:215-22.

22. Kauak L. Cirugía plástica post cirugía bariátrica. Revista Médica Clínica Las Condes. 2010;21:113-9.

23. Association WM. World Medical Association Declaration of Helsinki Ethical Principles for Medical Research Involving Human Subjects. JAME 2013;310:2191-4.

24. Sioka E, Tzovaras G, Katsogridaki G, Bakalis V, Bampalitsa S, Zachari E, et al. Desire for Body Contouring Surgery After Laparoscopic Sleeve Gastrectomy. Aesthetic Plast Surg. Springer US; 2015;39:1-7.
25. Shermak MA, Chang D, Magnuson TH, Schweitzer MA. An Outcomes Analysis of Patients Undergoing Body Contouring Surgery after Massive Weight Loss. Plastic and Reconstructive Surgery. 2006;118:1026-31.

26. Surgeons ASOP. 2016 Plastic Surgery Statistics Report. 2017 Jun pp. 1-25.

27. Chong T, Coon D, Toy J, Purnell C, Michaels J, Rubin JP. Body Contouring in the Male Weight Loss Population. Plastic and Reconstructive Surgery. 2012 Aug;130:325e-330e.

28. Aly A, Cram A, Chao M, Pang J, McKeon M. Belt Lipectomy for Circumferential Truncal Excess. Plastic and Reconstructive Surgery. 2003;111:398413.

29. Colwell A, Borud L. Optimization of Patient Safety in Postbariatric Body Contouring: A Current Review. Aesthet Surg J. 2008;28:437-42.

30. Danilla S, Domínguez C, Cuevas $P$, Calderón ME, Ríos MA, Andrades P, et al. The Body-QoL $\left({ }^{\circledR}\right)$ : patient reported outcomes in body contouring surgery patients [corrected]. Aesthetic Plast Surg. Springer US; 2014;38:575-83.

31. Danilla S, Cuevas P, Aedo S, Domínguez C, Jara R, Calderón ME, et al. Introducing the Body-QoL ${ }^{\circledR}$ : A New Patient-Reported Outcome Instrument for Measuring Body Satisfaction-Related Quality of Life in Aesthetic and Post-bariatric Body Contouring Patients. Aesthetic Plast Surg. Springer US; 2015;40:1-13.

32. Suijker J, Troncoso E, Pizarro F, Montecinos S, Villarroel G, Erazo C, et al. Long-Term Quality-of-Life Outcomes After Body Contouring Surgery: Phase IV Results for the Body-QoL ${ }^{\circledR}$ Cohort. Aesthet Surg J. 2017;42:1-10.

33. Klassen AF, Cano SJ, Scott A, Johnson J, Pusic AL. Satisfaction and Quality-ofLife Issues in Body Contouring Surgery Patients: a Qualitative Study. Obes Surg.
2012;22:1527-34.

34. van der Beek ESJ, Geenen R, de Heer FAG, van der Molen ABM, van Ramshorst B. Quality of Life LongTerm after Body Contouring Surgery following Bariatric Surgery. Plastic and Reconstructive Surgery. 2012;130:1133-9.

35. Kitzinger HB, Cakl T, Wenger R, Hacker S, Aszmann OC, Karle B. Prospective study on complications following a lower body lift after massive weight loss. British Journal of Plastic Surgery. Elsevier Ltd; 2013;66:231-8

36. Cardenas-Camarena LZ. Aesthetic Surgery of the Thoracoabdominal Area Combining Abdominoplasty and Circumferential Lipoplasty: 7 Years?? Experience. Plastic and Reconstructive Surgery. 2005;116:881-90.

37. Klopper EM, Kroese-Deutman HC, Berends FJ. Massive weight loss after bariatric surgery and the demand (desire) for body contouring surgery. Eur J Plast Surg. 2013;37:103-8.

38. Gmr RU, Banic A, Erni D. Is It Safe to Combine Abdominoplasty With Other Dermolipectomy Procedures to Correct Skin Excess After Weight Loss? Annals of Plastic Surgery. 2003;51:353-7.

39. Shermak MA, Rotellini-Coltvet LA, Chang D. Seroma Development following Body Contouring Surgery for Massive Weight Loss: Patient Risk Factors and Treatment Strategies. Plastic and Reconstructive Surgery. 2008;122:280-8.

40. Hurwitz D, Aghamohammadi S, Ota K, Unadkat J. A Clinical Review of Total Body Lift Surgery. Aesthet Surg J. 2008;28:294-303.

41. Andrades P, Prado A, Danilla S, Guerra C, Benitez S, Sepúlveda S, et al. Progressive Tension Sutures in the Prevention of Postabdominoplasty Seroma: A Prospective, Randomized, Double-Blind Clinical Trial. Plastic and Reconstructive Surgery. 2007;120:935-46. 